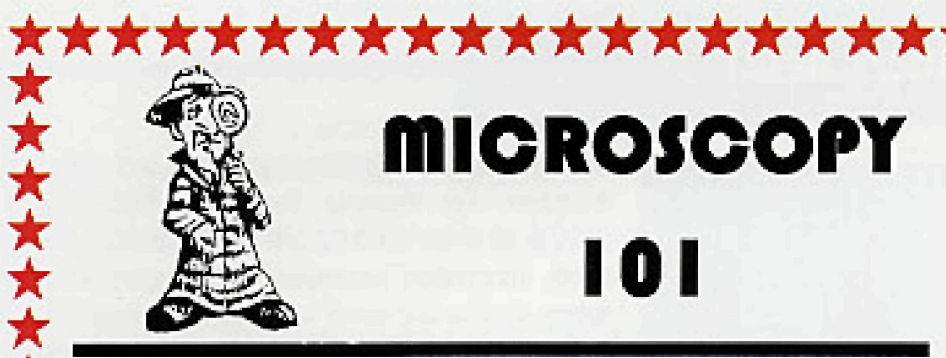

We appreciate the response to this publication feature - and welcome all contributions. Contributions may be sent to Phil Oshel, our Technical Edjtor at:

$\begin{array}{ll}\text { M. Phil Oshel } & \text { (608)B33-2885 } \\ \text { PO Box } 620068 & \text { eMail: oshelglenacom.net } \\ \text { Midolleton W } 53562 & \\ \text { (608)633-2885 } & \text { PLease note NEW address }\end{array}$

\section{A Hint For Reducing Background For Immuno-TEM}

Non-specific background staining is occasionally a problem with some antibodies and labeling systems. If nomal washing fails to reduce the backgound you might want to toy using a high salt buffer for washing. Phosphate buffered saline, or any other recipe with $\mathrm{NaCl}$ in it, is probably around $150 \mathrm{mM}(0.9 \%)$. Try boosting the NaCl to $5 \mathrm{X}$ normal. This would make it $750 \mathrm{mM}$, or $4.5 \%$ by weight.

If high salt buffer is used, remember to incubate the grids in a couple of changes of regular strength ( $150 \mathrm{mM}$ ) saline before going to the next step, to get the salt back into the range of physiologic strength.

An example would be to wash the grids on five drops of high salt buffer for about 2 minutes each, followed by two trops of nomal sall buffer for about 2 minutes each. Use this routine after incubating on the primary antibody or after any incubation you suspect may be contributing to background (secondary antibody, colloidal gold, etc.).

I have not found a good explanation for why this works, although it probably changes the conformation of proteins, alters their overall charge, and makes them less likely to bind to the surface of the sectioned material.

High salt concentrations are often used in biochemisty to precipitate proteins out of solution and to wash chromatography columns. Something similar is probably happening on the surface of the section, i. $e_{\text {., }}$ only those antibody molecules which have bound specifically to antigenic sites are able to stay on the section in the presence of high salt concentrations.

Robert (Bob) Chiovelti. E. Licht Company

\section{An Improved Fixation Method for Nerves}

The following fixation method is better than vascular perfusion for nerves from laboratory animals, and it might be adapled for biopsies.

1) Anaesthetize the animal and expose the part of the nerve to be fixed. While looking at the neve through a dissecting microscope, manipulate the limb (or other pert of the body) to stretch the nerve until its transverse striations (bands of Fontana) just disappear. (The bands of Fontana are due to undulations (waves) of the nerve fibres - $\mathrm{a}$ bit of microanatomy that was cleverly deduced before 1700 , long before the fibres themseves had been properly seen.)

2) Having straightened the nerve's fibxes in this way, drip the fixative (a buffered glutaraldehyde and formaldehyde mixture) onto the exposed nerve.

3) Apply a pellet of cotton wool soaked in the fixative, and wait for 30 to 60 minutes.

4) Remove the desired specimen and immerse it in fxative for a further 6 to 12 hours. Wash in buffer, post-osmicate, embed, section, stain (if desired), etc.

The superiority of this method over simple imimersion of perfusion was demonstrated by Moris ot. al. (1972), in one of four classical papers on the ultrastructure of the earliest stages of axonal regeneration. For the story of the bands of Fontana (a conspicuous but rarely discussed feature of nerve anatomy), see Hanicek 1986 (and also papers in J. Anat. by Beam \& others, 1970s). My second graduate student, Bruce Stelmack, was able to obtain useful measurements of external (with myelin) and external (axon) nerve fiber diameters in $4 \mu \mathrm{m}$ paraffin sections of facial and sciatic nerves of rats that had been fixed by Mortis ef. al., methods.

1. Hanicek, 1986. Analomy \& Embryology 174:407-411.

2. Morris, J H., A.R. Hudson, and C. Weddell. 1972. A study of degeneration and regeneration in the divided rat sciatic nerve based on electron microscopy, N. Changes in fascicular microtopography, perineurium and endoneurial fibroblasts, Zeitschrift fur Zelforschung 124:165-203. (This is THE classic in its field. Weddell was the boss. His work in the early $1940 \mathrm{~s}$ with J. Z. Young and W. Holmes provided the scientific foundation of modern peripheral neve repair surgery.)

Stelmack, B. M. and J. A. Kiernan. 1977. Effects of triodothyronine on the nomal and regenerating facial nerve of the rat. Acta Neuropathologica 40:151-155.

Jahn A. Kiernan, The University of Westem Ontario

\section{A Tip For Primary Antibody Elutlon:}

I have performed the erackation of the initial primary antibosy and its detection chemisty in the past and used a miciowave pepsin/HCl trealment after the first chromagen was developed. The procedure is detailed in Surgery, 1995. 118,957-66.

In this application we stained the same cells with two different cytoplasmic monoclonal antibodies (sequential immunohistochemical [IHC] staining). We had only one section to work with since the specimen was very limiled. The first antibody was stained and photographed, the chromagen was then removed (AEC), elution treatment was performed and the next antibody was stained. We ran a contol on another specimen and omitted the application of the second primary antibody, but applied all other detection reagents, and developed for the chromagen. These controls were consistently negetive, showing that the signal-generating complex had been removed by the elution or other unknown step.

To do it again, I would consider multicolor immunofluorescence.

A problem that I had with using Vector Red and AEC or Vector Red and DAB in simultaneous IHC staining was that the HRP chromagen covered up the Red - even under fuorescent excitation of the Vector Red. This is why we eventually tried a sequential format. If one is staining a nuclear and a cytoplasmic target most simultaneous systems work great, but when one is labeling two abundant cytoplasmic targets, problems can develop with chromagen overlapping. This is where I believe multicolor immunafluorescence would be great, because one could perform various wavelength excitations, or benefit by simultaneous blended colors.

Timothy Plummer, Mayo Clinic, Rochester, Minnesota

We Examine Carbon Nanotubes As Follows:

1) Add the specimen to a small volume of either acetone or methanol and "bial and error" will be needed to determine the exact dilution, so by different dilutions.

2) Suspend the sample by swirling (some people sonicate for several minutes) and use a pipette to tansfer a droplet onto a holey, filmed (Butuar or Fommar) and carbon coated grid (I suggest purchasing from commercial scurce).

3) Allow to air dy and examine in TEM looking for tubes suspended over the holes (best resolution)

The TEM should be set up for hi resolution with cooled taps over specimen area. A clean vacuum system is needed!

John J. Bozzola, Southem Illinois University 